\title{
Prevalência de álcool e drogas em mortes por afogamento no Rio Grande do Sul
}

\author{
C. S. Torres ${ }^{a,}$, M. D. Arbo ${ }^{a}$ \\ ${ }^{a}$ Faculdade de Farmácia, Universidade Federal do Rio Grande do Sul, Porto Alegre (RS), Brasil \\ *Endereço de e-mail para correspondência: carinetorres@igp.rs.gov.br Tel.: +55-51-991240846.
}

Recebido em 12/03/2019; Revisado em 24/04/2020; Aceito em 15/05/2020

\begin{abstract}
Resumo
O afogamento é a terceira causa mais frequente de mortes por traumatismo não intencional no mundo e sabe-se que, o consumo de álcool, drogas e fármacos são considerados fatores de risco associados ao afogamento. Neste contexto, o presente estudo visou avaliar a prevalência de álcool, drogas e fármacos em vítimas fatais de afogamento no Rio Grande do Sul no ano de 2015. Dados de afogamento e de análises toxicológicas sobre álcool, drogas e fármacos foram obtidos a partir do banco de dados do Departamento Médico Legal de Porto Alegre. Foram analisados 213 casos de afogamento e o álcool esteve presente em 37,55\% dos casos, já fármacos e drogas ilícitas em 12,6\%. O perfil epidemiológico aponta jovens do sexo masculino como principais vítimas de afogamento. A prevalência de álcool, drogas e fármacos pode ter contribuído para mortes por afogamento. Dessa forma, campanhas de prevenção, educação e conscientização da população poderiam resultar na redução dos índices de morte por afogamento.
\end{abstract}

Palavras-Chave: Afogamento, álcool, fármacos, drogas ilícitas.

\begin{abstract}
Drowning is the third most frequent cause of deaths from unintentional trauma in the world and it is known that the consumption of alcohol, drugs and pharmaceuticals are considered risk factors associated with drowning. In this context, the present study aimed to assess the prevalence of alcohol and drugs in drowning victims from Rio Grande do Sul in the year 2015. Data on drowning and toxicological analyzes on alcohol and drugs were obtained from the database of the Legal Medical Department of Porto Alegre. The toxicological results showed that alcohol was present in $37.55 \%$ of the cases, while drugs were detected in $12.6 \%$ of cases. The epidemiological profile pointed to young men as the main victims of drowning. The prevalence of alcohol was likely to have contributed to drowning deaths. Therefore, prevention and education campaigns could result in reduction of drowning rates.
\end{abstract}

Keywords: Drowning, alcohol, illicit drugs, drugs.

\section{INTRODUÇÃO}

A asfixia por afogamento é o processo de insuficiência respiratória por submersão ou imersão em líquido, geralmente de origem acidental. O corpo com sinais de afogamento pode apresentar externamente face cianótica, espuma bucal e nasal (cogumelo de espuma), pele anserina, lesões post-mortem por animais aquáticos. Já internamente o corpo pode exibir presença de líquido nas vias respiratórias, enfisema aquoso subpleural, hemorragias subpleurais (manchas de Paltauf), equimoses nos músculos e pescoço. Esta é uma importante causa de morbidade e mortalidade no mundo, contudo o afogamento ainda $\mathrm{e}$ pouco pesquisado no Brasil [1].
Em 2015, de acordo com estimativa da Organização Mundial da Saúde (OMS), 360.000 pessoas morreram por afogamento no mundo, o que torna um grande problema de saúde pública [2]. Nesse mesmo ano, os traumatismos que são lesões produzidas por agentes lesivos ou pela ação de energia externa, representaram mais de $9 \%$ da mortalidade mundial total. $\mathrm{O}$ afogamento, a qual é a terceira causa mais importante de mortalidade por traumatismo não intencional, representa $7 \%$ de todas as mortes relacionadas ao trauma [2].

No Brasil, no ano de 2010, foram registradas 1.198 internações hospitalares devido a esses acidentes, 63\% destes ocorridos na região Nordeste, com mortalidade em torno de $3 \%$ [3]. 
Entre as condições de risco para afogamento destaca-se o uso de álcool, que ao afetar o sistema nervoso central e os processos cognitivos, aumenta a probabilidade de exposição ao risco de asfixia. Segundo o Relatório Mundial sobre o Status do Álcool e Saúde de 2018 publicado pela Organização Mundial de Saúde, todos os anos há 3,3 milhões de mortes no mundo devido ao consumo prejudicial de álcool, representando $5,9 \%$ de todas as mortes. Estima-se também que 2,3 bilhões de pessoas consumam álcool atualmente no mundo [4]. Uma pesquisa conduzida na Suécia revelou que o álcool estava envolvido em $44 \%$ das mortes não intencionais por afogamento [5]. Percentuais similares, variando de aproximadamente 25 a $50 \%$, foram relatados em diferentes países como Irlanda e Hungria [6-7].

Por outro lado, há poucas informações disponíveis na literatura com relação ao papel de drogas ilícitas no caso de mortes por afogamento não intencional, além do álcool [8]. Os efeitos de muitas drogas psicotrópicas sobre a cognição e o julgamento são semelhantes aos efeitos produzidos pelo álcool no organismo $[9,10]$. Na Suécia, $22 \%$ dos óbitos entre vítimas não intencionais de afogamento morreram com a presença de uma ou mais drogas psicotrópicas constatadas no sangue, sendo os benzodiazepínicos e antidepressivos foram os principais fármacos encontrados [11].

Óbitos envolvendo histórico de afogamento são submetidos a exame necroscópico e à investigação toxicológica quanto ao uso de álcool e drogas, portanto oferece uma excelente oportunidade para investigar o papel de substâncias psicoativas nesse tipo de asfixia.

Dessa forma, o objetivo deste trabalho foi avaliar a prevalência de álcool e drogas nos casos de morte por afogamento no Estado do Rio Grande do Sul no ano de 2015, gerando novas informações para melhores ações de prevenção e conscientização.

\section{MATERIAIS E MÉTODOS}

\subsection{Coleta de dados}

Por lei, todas as mortes não naturais são submetidas à necropsia com a finalidade de determinar a causa mortis, sendo assim seu exame em casos de afogamento torna-se obrigatório e indispensável. Este estudo incluiu todos os casos de afogamento em que a necropsia foi realizada no Rio Grande do Sul no período de 1 de janeiro a 31 de dezembro de 2015. Os dados foram obtidos através do Protocolo Geral de Perícias (PGP) no Departamento Médico Legal (DML) e por meio de requisições encaminhadas ao Laboratório de Patologia Forense. A causa da morte foi presumida por achados da autopsia, informações policiais e achados microscópicos (presença de diatomáceas no pulmão). Pesquisas de plâncton confirmaram os achados da necropsia. As variáveis coletadas incluíram idade, sexo, mês, consumo de álcool e drogas pelas vítimas, região geográfica e Posto Médico Legal de atendimento.

A pesquisa de plâncton foi utilizada como referência de busca, uma vez que sempre são solicitados em casos suspeitos de afogamento conforme procedimento operacional padrão do DML. Dos 323 casos com solicitações microscópicas de plâncton, em 71 casos não houve solicitações toxicológicas, sendo estes excluídos do estudo. Além disso, 39 casos de óbitos indeterminados foram também excluídos (Fig. 1). O protocolo de pesquisa foi aprovado pelo Comitê de Ética e de Pesquisa da Universidade Federal do Rio Grande do Sul, sob o número do número do parecer 3.046.055 e CAAE 01907518.3.0000.5347.

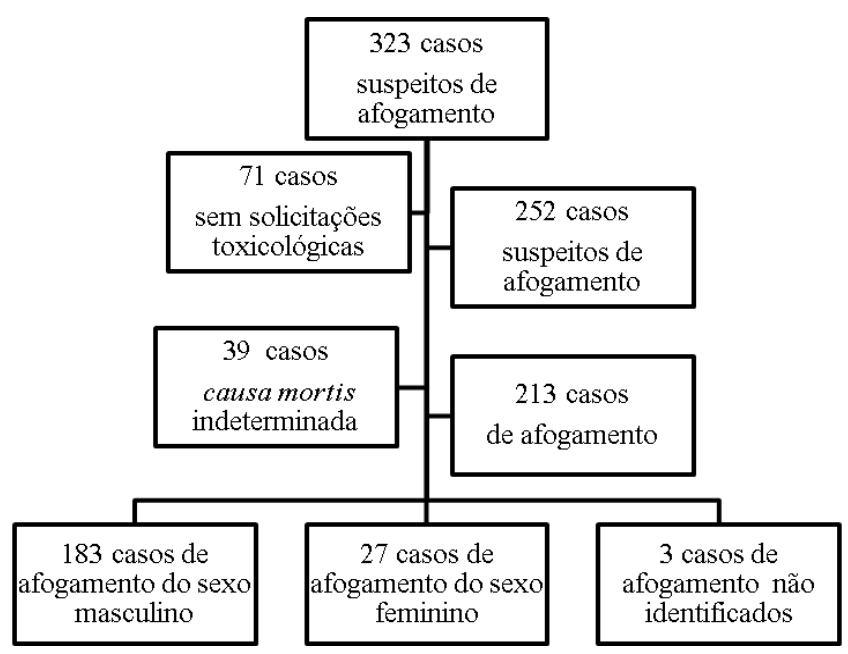

Figura 1. Diagrama do estudo

\subsection{Análise de plâncton}

O teste de diatomáceas consiste em analisar, em microscopia óptica, a presença de diatomáceas, em amostras coletadas de órgãos da vítima, após processos de degradação dos tecidos [12]. As informações sobre a pesquisa de plâncton foram colhidas do Laboratório de Patologia Forense do DML. As análises de plâncton foram realizadas em fragmento de pulmão submetidos a processamento histológico, seguido de microtomia $(20 \mu \mathrm{m}$ de espessura), desparafinização e montagem sem a utilização de coloração e detecção de diatomáceas por microscopia óptica de luz polarizada (Olympus, Tokio, Japão).

\subsection{Análise toxicológica}

Dados sobre álcool e drogas foram obtidos do Laboratório de Toxicologia Forense do Instituto Geral de Perícias. Análises de álcool foram realizadas em urina, humor vítreo e/ou sangue por cromatografia a gás com detector de ionização por chama e amostragem por headspace. Concentração de álcool no sangue (blood 
alcohol concentration, BAC), inferior a $3 \mathrm{dg} / \mathrm{L}$ foi considerada negativa no estudo, uma vez que baixas concentrações de etanol são mais prováveis de serem formadas por bactérias em decomposição [13].

Para pesquisa de psicotrópicos em fígado, o material foi submetido à extração com solventes orgânicos e posterior triagem toxicológica por cromatografia líquida de alta eficiência com detector de arranjo de diodos (CLAE-DAD) para os seguintes grupos de substâncias: antidepressivos tricíclicos (amitriptilina, clomipramina, imipramina e nortriptilina), benzodiazepínicos (bromazepam, clonazepam, diazepam, flunitrazepam e midazolam), drogas básicas (cocaína), fenotiazínicos (clorpromazina, levomepromazina, periciazina e prometazina), hidantoínas(fenitoína), opioides (codeína, morfina e petidina), barbitúricos (barbital e fenobarbital) e outros (ácido valpróico, carbamazepina, citalopram, fluoxetina, lidocaína, 3,4-metilenodioximetanfetamina - MDMA e sertralina).

Amostras de urina foram encaminhadas à triagem toxicológica por imunoensaio e, quando aplicável, à análise confirmatória por cromatografia. Foram testados o(s) seguinte(s) grupo(s) de substância(s) com atividade psicotrópica: canabinoides (11-nor-9-carbóxi-THC), cocaína e/ou metabólitos (cocaína e éster de metilecgonina), anfetamínicos (anfetamina, metanfetamina, metilenodioxianfetamina - MDA e MDMA) e benzodiazepínicos e produtos de biotransformação (2-amino-5-bromofenil) (2-piridil) metanona (ABP), 2-amino-5-clorobenzofenona (ACB), 2amino-5-cloro-2'-fluorbenzofenona (ACFB), 2-amino2',5-diclorobenzofenona (ADB), 2-amino-2'-cloro-5nitrobenzofenona (ANCB), 5-cloro-2 (metilamino) benzofenona (MACB) e 2'-fluor-2-(metilamino)-5nitrobenzofenona (MNFB).

\section{RESULTADOS E DISCUSSÕES}

\subsection{Afogamento e regiões}

Durante o período do estudo, 323 mortes relacionadas com afogamento foram periciadas. O serviço de medicina legal é exercido na capital e no interior do Estado por postos localizados em diferentes regiões do Rio Grande do Sul de acordo com a demanda e necessidade. Porto Alegre foi responsável por autopsiar cerca de 19,19\% dos casos, conforme Fig. 2, índice que se relaciona com a região de maior número de óbitos, conforme Fig. 3. Observa-se também um elevado número de perícias no Posto Médico Legal (PML) de Osório, cidade que compreende os atendimentos do litoral. A desproporção na incidência de afogamento deve-se a diferenças na demografia, geografia, medidas preventivas e comportamento de risco [14]. Além disso, por conter uma maior extensão de praias, e concentrar o maior número de banhistas, naturalmente, as ocorrências de afogamento possuem maior incidência na região litorânea [15].

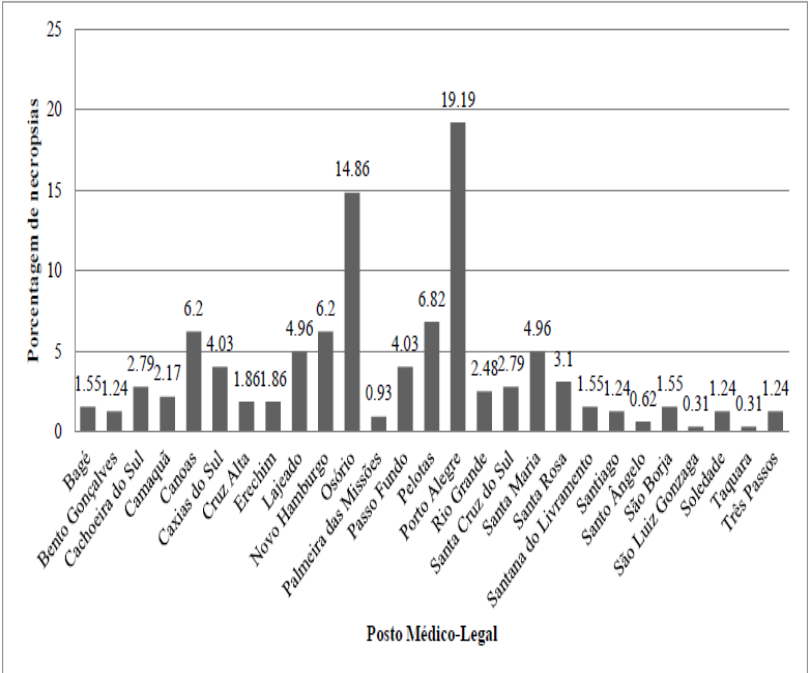

Figura 2. Número de perícias de óbitos suspeitos por afogamento por Posto Médico-Legal registrado no ano de 2015 no Rio Grande do Sul $(n=323)$.

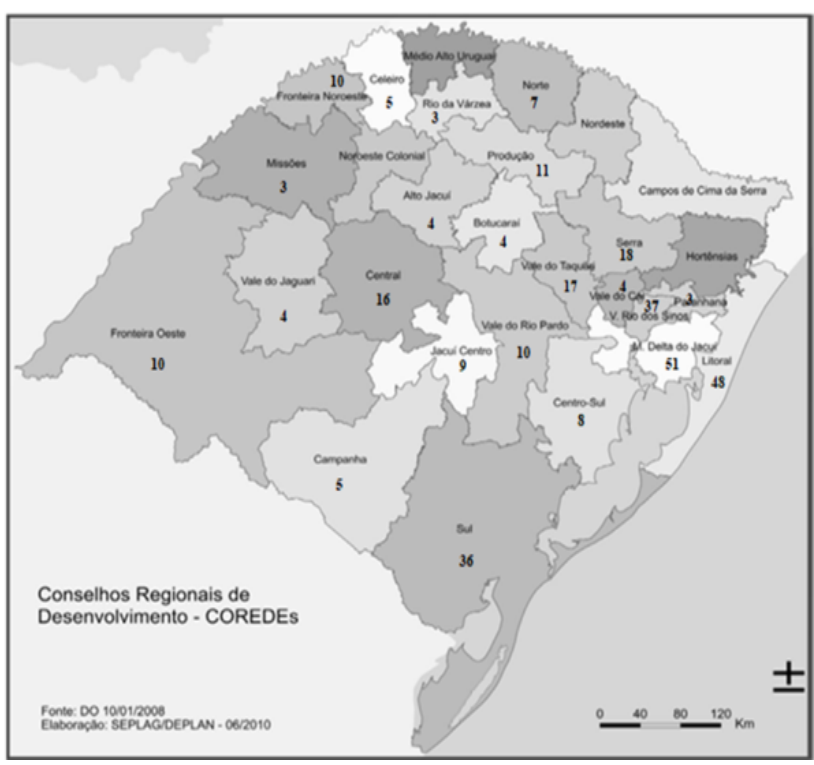

Figura 3. Número de óbitos suspeitos por afogamento por região registrado no ano de 2015 no Rio Grande do Sul (n=323). (Fonte: PRÓRS IV - propostas estratégicas para o desenvolvimento regional no Estado do Rio Grande do Sul, no período 2011 - 2014).

\subsection{Afogamento e temporada}

A maior prevalência de mortes por afogamento foi verificada nos meses de verão, com pico no mês de janeiro $(19,24 \%)$ (Fig. 4). Mortes por afogamento no outono e inverno não são insignificantes, entretanto a elevada temperatura nos meses de verão e o período de férias contribuem para o aumento de atividades aquáticas e banhos de mar, rios ou lagos, aumentando a incidência de afogamentos no verão. O padrão sazonal de mortes por afogamento foi semelhante em comparação a estudos de outros países como Austrália e Hungria [7-16]. 


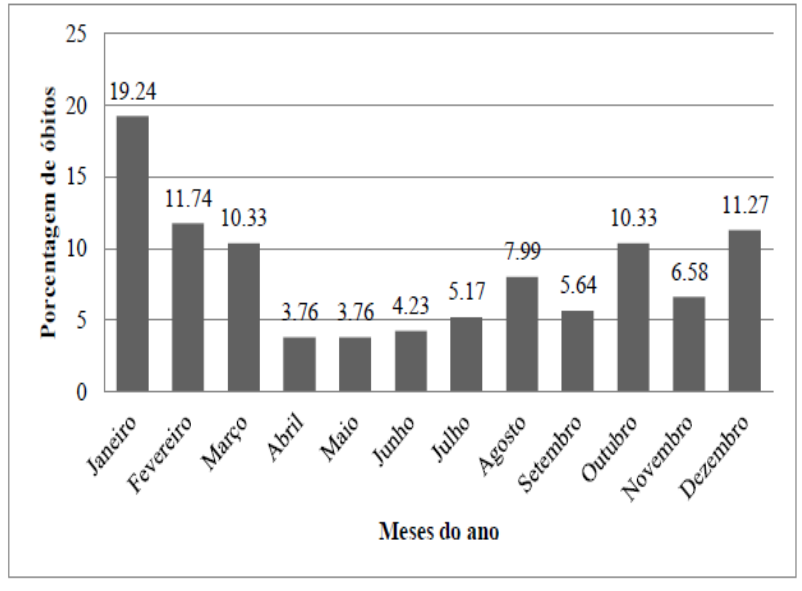

Figura 4. Número de óbitos por afogamento em relação aos meses registrados no ano de 2015no Rio Grande do Sul (n=213).

\subsection{Afogamento e idade}

Do total de 252 mortes por afogamento, a faixa etária de 10-19 anos apresentou maior ocorrência, com um total de 38 mortes $(15,07 \%)$, corroborando com o estudo realizado em Salvador/BA [17]. Tal fato pode ser explicado pela falta de habilidade de natação, falta de discernimento ou realização de atividades aquáticas desacompanhado de adultos [11]. Por outro lado, em países, como Hungria, o número de mortes por afogamento foi maior entre as idades 51 e 60 anos [7], mesma faixa etária semelhante à observada na Suécia, que demonstrou predomínio de mortes do sexo masculino com média de idade de 53 anos [11]. Diferentemente do encontrado no Rio Grande do Sul, grupos etários mais velhos ainda têm prevalência moderadamente alta de afogamento em diversos países, explicado por uma pre-venção mais efetiva para grupos etários mais jovens [11]. Observa-se também uma elevada taxa de idade não disponível no banco de dados e nas requisições encaminhadas ao laboratório de patologia forense (Tab. 1).

\subsection{Afogamento e sexo}

Outras pesquisas disponíveis [5-7-11-15-16] registram índices maiores de afogamento entre homens do que mulheres. Situação observada também no Rio Grande do Sul, na qual houve predominância do sexo masculino, sendo constatadas 183 mortes por afogamentos $(85,91 \%)$. Assim, atribui-se ao homem uma personalidade mais agressiva e aventureira, superestimando sua capacidade natatória [18].

\subsection{Afogamento e pesquisa de plâncton}

Os resultados de pesquisa de plâncton (Tab. 2) apresentaram positividade em 157 casos (73,70\%), entretanto deve-se levar em consideração que pode ocorrer à possibilidade de contaminação, com introdução de diatomáceas externas, contidas nos reagentes ou instrumentos da mesa de autópsia [19]. Além disso, a morte por afogamento nem sempre é decorrente de aspiração maciça de água. Nos casos em que o resultado foi negativo (7 resultados), deve-se levar em consideração que podem ocorrer espasmos da laringe, ocasionando a liberação de um muco espesso, que acaba obstruindo as vias aéreas (afogamento seco) [20-21]. Outra forma é a ocorrência de hiperventilação, provocando a inalação de água de modo prematuro, levando a uma morte mais rápida, com menor volume de água [20-21]. Nestes casos, pode-se não verificar a presença de diatomáceas sendo necessários métodos mais modernos de investigação, como PCR (Polymerase Chain Reaction) [20].

Tabela 1. Número de óbitos por afogamento por faixa etária registrado no ano de 2015 no Rio Grande do Sul (n=252).

\begin{tabular}{cccc}
\hline Idade & $\begin{array}{c}\text { Números de óbitos por } \\
\text { afogamento Masculino }\end{array}$ & $\begin{array}{c}\text { Números de óbitos por } \\
\text { afogamento Feminino }\end{array}$ & $\begin{array}{c}\text { Número de óbitos de } \\
\text { causa indeterminada }\end{array}$ \\
$\mathbf{0 - 9}$ & 3 & 3 & 2 \\
$\mathbf{1 0 - 1 9}$ & 34 & 2 & 2 \\
$\mathbf{2 0 - 2 9}$ & 27 & 2 & 6 \\
$\mathbf{3 0 - 3 9}$ & 19 & 2 & 3 \\
$\mathbf{4 0 - 4 9}$ & 25 & 1 & 8 \\
$\mathbf{5 0 - 5 9}$ & 22 & 4 & 6 \\
$\mathbf{6 0 - 6 9}$ & 13 & 3 & 4 \\
$\mathbf{7 0 - 7 9}$ & 5 & 2 & 1 \\
$\mathbf{8 0 - 8 9}$ & 2 & 1 & 1 \\
$\mathbf{> 9 0}$ & 1 & - & - \\
\hline Não disponível & 32 & 7 & 6 \\
\hline Total & $\mathbf{1 8 3}$ & $\mathbf{2 7}$ & $\mathbf{3 9}$ \\
\hline
\end{tabular}

*3 casos de afogamento indeterminado sem idade disponível. 
Tabela 2. Números de pesquisa de plâncton em casos de morte por afogamento no ano de 2015 no Rio Grande do Sul.

\begin{tabular}{cccc}
\hline & Pesquisa de Plâncton & & \\
Sexo & Positivos & Negativos & Prejudicados \\
Masculino & 129 & 7 & 47 \\
Feminino & 26 & - & 1 \\
Não identificado & 2 & - & 1 \\
Total & 157 & 7 & 49 \\
\hline
\end{tabular}

Além disso, diatomáceas podem estar ausentes nos afogamentos em água doce, justamente pela sua baixa ou nenhuma concentração encontrada na água. Apesar de apresentar resultados negativos não se descarta o óbito por afogamento, uma vez que foram encontrados sinais característicos de afogamento no exame de necropsia.

O estudo apresentou 49 exames $(23,0 \%)$ com material inadequado para análise. A falta do preparo pré-analítico favorece a ocorrência de material autolisado, uma vez que as peças anatômicas devem ter tamanho pequeno e devem ser acondicionadas em frascos que contenham formol a $10 \%$, sendo o volume de formol 10 vezes o da peça para uma boa fixação [21]. Outro fator que prejudica o resultado é o estado de decomposição do cadáver, uma vez que o material coletado originalmente já se encontra autolisado.

\subsection{Afogamento e consumo de álcool e drogas}

\subsubsection{Afogamento e consumo de álcool}

Em pesquisa realizada na Suécia e Austrália [11-16] indicam que o álcool é um grande fator contribuinte para mortes por afogamento. No estudo, das 213 mortes confirmadas por afogamento em que foram realizadas análises toxicológicas somente 2 amostras testadas apresentaram positividade em urina e humor vítreo. Já a concentração de álcool no sangue foi positiva em 98 (46,0\%) de 213 casos de afogamento (Tab. 3).

Nesse caso, a concentração de álcool no sangue

(blood alcohol concentration, BAC) $\geq 3 \mathrm{dg} / \mathrm{L}$ estava presente em 78 indivíduos com um teor médio de alcoolemia de 16,42 $\pm 11,32 \mathrm{dg} / \mathrm{L}$. O nível de álcool no sangue de vítimas do sexo masculino foi superior ao sexo feminino. Na Suécia, uma proporção de $44 \%$ de casos positivos para álcool no sangue foi demonstrada em casos de afogamento [11]. Já em cinco grandes cidades na África do Sul, o álcool foi detectado em $40 \%$ dos casos [22].

No tráfego rodoviário é bem comprovado que o álcool prejudica os motoristas em muitos aspectos, incluindo atitude, julgamento, vigilância, percepção, reação e controle [23]. À medida que o BAC aumenta, a qualidade do julgamento e da restrição deteriora-se rapidamente [11]. Fica evidente no afogamento que o álcool não influencia somente a tomada de decisão, mas também reduz a consciência dos cuidados necessários para evitar acidentes, e, por sua vez, a capacidade de salvar-se, uma vez que está em dificuldade.

\subsubsection{Afogamento e uso de fármacos e drogas ilícitas}

A correlação de álcool e afogamento foi demonstrada em diversas pesquisas [7,12], entretanto o efeito da contribuição de drogas psicotrópicas ainda é um assunto pouco conhecido. Em estudo realizado na Suécia, 22\% dos casos foram positivos para drogas psicotrópicas [11]. Em pesquisa realizada nos EUA, foi demonstrado que $31 \%$ dos casos eram negativos para álcool, mas positivos para outras drogas, contudo não havia dados individuais para drogas psicotrópicas. Em ambos os estudos, os casos foram apresentados apenas como droga positiva ou droga negativa, sem concentrações dos diferentes tipos de substâncias [24].

Em nosso estudo, análises toxicológicas identificaram fármacos em 5,6\% dos casos (8 homens e 4 mulheres) (Tab. 4), as mais comumente encontradas foram as moléculas ACB que estão relacionada ao uso de camazepam, cetazolam, clordiazepóxido, clorazepato, diazepam, halazepam, medazepam, nordazepam, oxazepam, oxazolam, pinazepam, prazepame/ou temazepam e a MACB, relacionada ao uso de camazepam, diazepam, cetazolam e/ou temazepam (Tab. 5).

Uma pesquisa realizada pelo Centro Brasileiro de Informações sobre Drogas (CEBRID) em parceria com a Universidade Federal de São Paulo (UNIFESP) em 2005 informou que no Brasil ocorreu um aumento do uso na vida (uso de droga pelo menos uma vez na vida) de benzodiazepínicos, de 3,3\% em 2001 para 5,6\% em 2005 [25]. Fármacos como benzodiazepínicos utilizados para diminuir a ansiedade, possuem como efeitos indesejáveis sonolência, comprometimento cognitivo e alteração na habilidade psicomotora, tais efeitos adversos devem ser levados em consideração em relação a atividades recreativas em ambientes aquáticos [24].

Drogas ilícitas foram encontradas em 7,04\% dos casos de afogamento (Tab.5) sendo a cocaína a droga mais observada (12 casos), identificada pela presença do éster de metilecgonina, um dos seus metabolitos. A cocaína causa uma excitação geral do organismo, os usuários sentem geralmente uma sensação de poder, força e euforia, mas o indivíduo também fica irrequieto, trêmulo e impaciente [24]. De acordo com o II Levantamento 
Domiciliar sobre o uso de drogas psicotrópicas no Brasil, realizado pelo CEBRID e UNIFESP em 2005, foi relatado que na região Sul houve aumento do uso na vida de maconha, solventes, estimulantes, opiáceos, alucinógenos, crack, esteroides, merla e heroína [25].

A região Sul aparece em segundo lugar em prevalência de maior uso na vida de cocaína (3,1\%). O II Levantamento Nacional de Álcool e Drogas (LENAD) de 2012 aponta a cocaína como segunda droga de prevalência de uso na população brasileira. [26]. Já em Porto Alegre, a pesquisa realizada sobre o perfil dos usuários que acessam os Centros de Atenção Psicossocial para Álcool e outras Drogas (CAPS AD), informa que a droga de prevalência foi o álcool, seguida do crack e cocaína [27]. O estudo ainda relata que os usuários são predominantemente homens, adultos, concentrados na faixa etária dos 30 aos 50 anos, com baixa renda e ensino fundamental incompleto. Assim, esses elementos corroboram para a prevalência encontrada em nosso estudo. Segundo pesquisa realizada no Brasil [28], o uso de cocaína é mais comum entre adolescentes e jovens adultos do sexo masculino, fatores que indicariam a negatividade encontrada nas análises de drogas ilícitas em vítimas do sexo feminino.

A presença de tetraidrohidrocanabinol (THC), o principal componente psicoativo da cannabis, foi identificada pela presença do metabólito canabinoide 11-nor-9-carbóxiTHC. Os dados do uso na vida de maconha, em 2001, foram de $6,9 \%$ aumentando para $8,8 \%$ em 2005 e o uso de maconha é maior para o sexo masculino quando comparado ao feminino [25]. Os sintomas relacionados ao consumo de cannabis incluem despersonalização, desrealização, paranoia, ideias de referência, fuga de ideias, pensamento acelerado, pensamento desorganizado, delírios persecutórios, delírios de grandeza, alucinações auditivas e visuais, e prejuízos na atenção e memória em uma consciência de outra forma clara [29].

As manifestações descritas em ambas as drogas contribuem para comportamento de risco, alterando a percepção da realidade, o que pode resultar em acidente com morte por afogamento. Essas drogas podem afetar o discernimento e a consequente possibilidade de sobrevivência após a queda do usuário na água [30].

\subsection{Afogamento e associação de álcool e drogas}

A combinação de álcool com fármacos e drogas ilícitas esteve presente em 4 e 9 casos, respectivamente (Tab. 6). $\mathrm{Na}$ associação de benzodiazepínicos e álcool, por exemplo, o etanol aumenta a depressão do sistema nervoso central e o risco de sobredosagem [31].

A combinação de álcool e cocaína é considerada uma associação frequente em usuários de drogas, o consumo simultâneo de cocaína e álcool tem aumentado significativamente nos últimos anos em todo o mundo [32]. A interação metabólica do etanol e da cocaína origina um novo metabólito, chamado cocaetileno, que desempenha um papel importante no aumento do prazer e na toxicidade derivada do consumo de ambas as drogas. Isso produz um aumento considerável de euforia e sua duração, bem como uma diminuição da disforia e abstinência à cocaína, juntamente com um consumo mais compulsivo [33]. Estudo em modelo animal também relata que o álcool interage sinergicamente com a cocaína [34]. Nesse sentido, estudo realizado em 2013 [35] menciona que o cocaetileno causaria o benefício de levar um tempo maior para ser eliminado (tempo de meia-vida mais longo).

Tabela 3. Presença de álcool nos casos de morte por afogamento no ano de 2015 no Rio Grande do Sul.

\begin{tabular}{|c|c|c|c|c|c|c|}
\hline \multicolumn{7}{|c|}{ Sexo } \\
\hline *Álcool & Masculino & $\%$ & Feminino & $\%$ & Não identificado & $\%$ \\
\hline Positivo & $* * 91$ & 42,72 & 8 & 3,76 & 1 & 0,47 \\
\hline Negativo & 75 & 35,22 & 17 & 7,99 & 1 & 0,47 \\
\hline Prejudicado & 5 & 2,34 & - & - & - & - \\
\hline Não testado & 12 & 5,63 & 2 & 0,94 & 1 & 0,47 \\
\hline Total & 183 & 85,91 & 27 & 12,69 & 3 & 1,41 \\
\hline \multicolumn{7}{|l|}{$\mathrm{BAC}(\mathrm{dg} / \mathrm{L})$} \\
\hline$<3$ & & 17 & 3 & & - & \\
\hline 3-4 & & 10 & 1 & & - & \\
\hline $5-9$ & & 14 & 1 & & - & \\
\hline 10-14 & & 8 & 1 & & - & \\
\hline 15-19 & & 11 & - & & 1 & \\
\hline $20-24$ & & 7 & - & & - & \\
\hline 25-29 & & 12 & - & & - & \\
\hline 30-34 & & 6 & 1 & & - & \\
\hline$\geq 35$ & & 4 & 1 & & - & \\
\hline Testado em humor vítreo & & 1 & - & & - & \\
\hline Testado em urina & & 1 & - & & - & \\
\hline Total & & 91 & 8 & & 1 & \\
\hline
\end{tabular}


Tabela 4. Presença de fármacos e drogas ilícitas nos casos de morte por afogamento no ano de 2015 no Rio Grande do Sul (n=2013).

\begin{tabular}{|c|c|c|c|c|c|c|}
\hline \multicolumn{7}{|c|}{ Sexo } \\
\hline Fármacos & Masculino & $\%$ & Feminino & $\%$ & $\begin{array}{c}\text { Não } \\
\text { identificado }\end{array}$ & $\%$ \\
\hline Positivo & $7(1)^{*}$ & 3,75 & 4 & 1,88 & - & - \\
\hline Negativo & 154 & 72,30 & 15 & 7,05 & 3 & 1,41 \\
\hline Prejudicado & 1 & 0,47 & - & - & - & - \\
\hline Não testado & 20 & 9,39 & 8 & 3,76 & - & - \\
\hline Total & 183 & 89,91 & 27 & 12,69 & 3 & 1,41 \\
\hline \multicolumn{7}{|c|}{ Drogas ilícitas } \\
\hline Positivo & \multicolumn{2}{|r|}{$14(1)^{*}$} & \multicolumn{2}{|c|}{-} & \multicolumn{2}{|l|}{1} \\
\hline Negativo & \multicolumn{2}{|r|}{147} & \multicolumn{2}{|c|}{19} & \multicolumn{2}{|l|}{2} \\
\hline Prejudicado & \multicolumn{2}{|r|}{1} & \multicolumn{2}{|c|}{-} & \multicolumn{2}{|l|}{-} \\
\hline Não testado & \multicolumn{2}{|r|}{20} & \multicolumn{2}{|c|}{8} & \multicolumn{2}{|l|}{ - } \\
\hline Total & \multicolumn{2}{|r|}{183} & \multicolumn{2}{|c|}{27} & \multicolumn{2}{|l|}{3} \\
\hline
\end{tabular}

*Uma amostra apresentou positividade para fármacos e drogas.

Tabela 5. Tipos de fármacos e drogas ilícitas nos casos de morte por afogamento no ano de 2015 no Rio Grande do Sul.

\begin{tabular}{lc}
\hline Tipos & Quantidade \\
\hline Benzodiazepínicos & 5 \\
Associação de & 1 \\
$\begin{array}{l}\text { Benzodiazepínicos e } \\
\text { cocaína }\end{array}$ & \\
Ácido valproico & 1 \\
Carbamazepina & 1 \\
Prometazina & 1 \\
Fenobarbital & 1 \\
Antidepressivos & 2 \\
tricíclicos & \\
THC & 3 \\
Associação de THC e & 4 \\
cocaína & \\
Cocaína & 7 \\
Total & $\mathbf{2 6}$ \\
\hline
\end{tabular}

O uso concomitante de cannabis e álcool é igualmente comum. Segundo diferentes estudos, a prevalência está entre 20-34\%, dependendo das diferentes amostras estudadas [36]. O nível de THC detectado no sangue é maior quando o THC é consumido com álcool, quando comparado a cannabis consumida sozinha [37]. O mesmo estudo relata que a direção veicular foi mais prejudicada nas combinações de THC e álcool, o que deve se refletir também nos reflexos natatórios.

\subsection{Outras associações}

Observou-se também a associação de THC e cocaína em dois casos, os quais foram negativos para álcool. Estudos demonstram que usuários constantes de cannabis são sensíveis ao estímulo e inibição dos efeitos da cocaína na função psicomotora e impulsividade, respectivamente. A redução na proficiência do controle dos impulsos observada durante a intoxicação por THC e cocaína pode colocar os usuários de drogas em risco de uso repetido de drogas e dependência [38]. Portanto, a associação de cannabis e cocaína levariam a um aumento no comportamento de risco, proporcionando perigo aos usuários.

\section{LIMITAÇÕES}

O presente estudo foi baseado em registros coletados em banco de dados e requisições, sendo assim informações sobre circunstancias do afogamento não foram obtidos. Dessa forma, não é possível analisar separadamente mortes por afogamento intencionais de não intencionais. Em 71 casos não houve solicitações toxicológicas, portanto essa sub-representação de mortes por afogamento pode limitar a precisão das análises.

Tabela 6. Associação de álcool, fármacos e drogas nos casos de morte por afogamento no ano de 2015 no Rio Grande do Sul (n=26).

\begin{tabular}{|c|c|c|c|c|c|c|}
\hline \multicolumn{7}{|c|}{ Sexo } \\
\hline Associação & Masculino & $\%$ & Feminino & $\%$ & Não identificado & $\%$ \\
\hline Álcool + fármacos & 4 & 15,38 & - & - & - & - \\
\hline Álcool + drogas ilícitas & 8 & 30,76 & - & - & 1 & 3,84 \\
\hline Drogas ilícitas & 7 & 26,92 & - & - & - & - \\
\hline Fármacos & 2 & 7,69 & 4 & 15,38 & - & - \\
\hline Total & 21 & 80,75 & 4 & 15,38 & 1 & 3,84 \\
\hline
\end{tabular}


Dados sobre concentrações de álcool no sangue total devem ser analisados com cuidados. Além da atividade microbiana que interfere na dosagem do álcool, a concentração de etanol varia de acordo com o local onde foi realizada a coleta de sangue, gerando complicações na interpretação dos resultados. Por norma [20], a coleta deve ser realizada por ordem de preferência, se possível, da veia femoral, veia subclávia e coração, contudo não se descarta a coleta em diferentes regiões. Além disso, não é possível descartar que níveis elevados de BAC possam indicar tentativa de suicídio.

Em pesquisa realizada na Suécia demonstrou que drogas psicoativas estavam presentes em $71 \%$ dos suicídios envolvendo afogamento [11]. Desse modo, a utilização de álcool em associação ou não com substâncias psicotrópicas como via de suicídio não pode ser rejeitada. Portanto, são necessários estudos futuros para confirmar o papel de álcool e drogas em mortes por afogamento intencionais e não intencionais.

\section{CONCLUSÃO}

$\mathrm{O}$ risco de morte por afogamento envolve inúmeros fatores, entre eles destaca-se o consumo de álcool como fator precursor de mortalidade. Em relação ao perfil das vítimas, houve predomínio de jovens na faixa etária de 1019 anos, sexo masculino, bem como as maiores ocorrências de afogamento nos meses de verão. O álcool esteve presente em aproximadamente $37,55 \%$ dos casos de afogamento, índice próximo ao encontrado em diferentes países. Já fármacos e drogas ilícitas estiveram presentes em aproximadamente $12,64 \%$ dos casos.

Embora a prevalência de fármacos e drogas ilícitas seja baixa, é importante salientar que ambas contribuem para um comportamento de risco quando utilizadas sozinhas ou em associação com o álcool. Portanto, estratégias de prevenção e educação que minimizem o uso abusivo de álcool e drogas são recomendadas. O conhecimento dos fatores de risco se torna essencial para a redução da mortalidade por afogamento.

\section{REFERÊNCIAS BIBLIOGRÁFICAS}

[1] D. Szpilman. Afogamento. Rev. Bras. Med. Esporte6: 131-144 (2000).

[2] WHO. Ahogamientos. Disponível em: $<\mathrm{http}: / / \mathrm{www}$. who.int/es/news-room/fact-sheets/detail/drowning>.

Acesso em: 29 jun. 2018.

[3] F. M. L. P. Júnior; E. M. C. Tabosa; M. C. C. Viana. Perfil clínico e epidemiológico de pacientes vítimas de afogamento no município de Fortaleza/CE. Rev. Saúde Públ. 5: 19-27 (2012).

[4] WHO. Alcohol. Disponível em: http://www.who.int/ es/news-room/fact-sheets/detail/alcohol>. Acesso em: 22 nov. 2018.
[5] T. R. Driscoll; J. A Harrison; M. Steenkamp. Review of the role of alcohol in drowning associated with recreational aquatic activity. Inj. Prev 10: 107-113 (2004).

[6] H. Ingoldsby; G. Callagy. Alcohol and unnatural deaths in the West of Ireland: a 5-year review. J. Clin. Pathol. 63:900-903 (2010).

[7] E. Rácz; F. Könczöl; H. Mészáros; Z. Kozma; M. Mayer; Z. Porpáczy; V. S. Poór; K. Sipos. Drowningrelated fatalities during a 5-year period (2008-2012) in South-West Hungary--a retrospective study.J. Forensic Leg. Med. 31: 7-11 (2015).

[8] OH. Drummer. Benzodiazepines - effects on human performance and behavior. Forensic Sci Rev. 14:1-14 (2002).

[9] I. Amado-Boccara. Effects of antidepressants on cognitive functions: a review. Neurosci Biobehav Ver. 19:479-493 (1995).

[10] S.Y Leung. Benzodiazepines, opioids and driving: an overview of the experimental research. Drug Alcohol Rev. 30:281-6 (2011).

[11] K. Ahlm; B. Saveman; U. Björnstig. Drowning deaths in Sweden with emphasis on the presence of alcohol and drugs - a retrospective study, 1992-2009. BMC public health. 13: 216 (2013).

[12] L. Donadel; N. Cardoso; A. Hoenisch; L. R. P. Utz. Revisão sobre o diagnóstico de afogamento com o uso do plâncton, teste de diatomáceas e de PCR. Rev. Bras. Crim. 3: 17-23 (2014).

[13] F. C. Kugelberg; A. W. Jones. Interpreting results of ethanol analysis in postmortem specimens: a review of the literature. Forensic Sci. Int. 165: 10-29 (2007).

[14] J. Howland; R. Hingson; T. W. Mangione; N. Bell; S. Bak. Why are most drowning victims men? Sex differences in aquatic skills and behaviors. Am. J. Public Health 86: 93-6 (1996).

[15] G. A. Gomes; D. Biffi; V. R. Ribeiro. Perfil epidemiológico das vítimas de afogamento do estado do Rio Grande do Sul. R. Perspect. Ci. e Saúde 2: 13-22 (2017).

[16] A. E. Peden; R. C. Franklin; P. A. Leggat. Alcohol and its contributory role in fatal drowning in Australian rivers, 2002-2012.Accid. Anal. Prev. 98: 259-265 (2017).

[17] M. P. Silva. Panorama das mortes por afogamento de crianças e adolescentes em Salvador, Bahia (Brasil). Trabalho de Conclusão de Curso. Faculdade de Medicina, Universidade Federal da Bahia (2015).

[18] A. S. S. Segundo; M. C. Sampaio. Perfil epidemiológico dos afogamentos em praias de Salvador, Bahia. Epidemiol. Serv. Saúde. 24:31-38 (2015)

[19] B. A. C. Faria. Diatomáceas no contexto da investigação das mortes por afogamento. Tese de Mestrado. Departamento de Medicina Legal e Ciências Forenses, Faculdade de Medicina da Universidade de Coimbra (2013).

[20] Elaboração do Procedimento Operacional Padrão sobre exame necrológico e exames realizados do 
Departamento Médico Legal. Rio Grande do Sul: Instituto Geral de Perícias (2014).

[21] F. S. Benfica; M. Vaz; M. Rovinski; M. S. T. B. Costa. Manual Atualizado de Rotinas do Departamento Médico-Legal do Estado do Rio Grande do Sul, Brasil (2015).

[22] H. Donson; A. V. Niekerk. Unintentional drowning in urban South Africa: a retrospective investigation, 2001-2005. Int. J. Inj. Control Saf. Promot. 20: 218-226 (2013).

[23] X. Zhao; X. Zhang; J. Rong. Study of the effects of alcohol on drivers and driving performance on straight road. Math. Probl. Eng. 2014: 1-9 (2014).

[24] T. Pajunen; E. Vuori; F F. Vincenzi; P. Lillsunde; G. Smith; P. Lunetta Unintentionaldrowning: Role of medicinal drugs and alcohol.BMC Public Health. 17:388 (2017).

[25] E.A. Carlini; J.C. Galduróz; A.R. Noto; C.M. Carlini; L.G. Oliveira; S.A. Nappo; Y.G. Moura; Z.V.D.M. Sanchez. II Levantamento Domiciliar Sobre o Uso de Drogas Psicotrópicas no Brasil: estudo envolvendo as 108 maiores cidades do país: 2005, Brasil (2007).

[26] R. Laranjeira. II Levantamento Nacional de Álcool e Drogas: 2012, Brasil (2014).

[27] L. S. Leite; M. A. P. Oliveira; S. J. E. Santos. Perfil dos usuários dos centros de atenção psicossocial para álcool e outras drogas de Porto Alegre: um estudo preliminar. Bol. da Saúde. 25:121-131 (2016).

[28] L. B. Dualibi; M. Ribeiro; R. Laranjeira. Perfil dos usuários de cocaína e crack no Brasil. Cad. de Saúde Pública. 24:545-557 (2008).

[29] R. A. Sewell; P. D. Skosnik; I. Garcia-Sosa; M. Ranganathan; D. C. D'Souza. Efeitos comportamentais, cognitivos e psicofisiológicos dos canabinoides: relevância para a psicose e a esquizofrenia. Rev. Bras. Psiquiatr. 32: 515-530 (2010).

[30] A. Engeland; S. Skurveit; J; Mørland. Risk of road traffic accidents associated with the prescription of drugs: a registry-based cohort study. Annals of Epidemiology.

17: 597-602 (2007).

[31] C. Jones; L. Paulozzi; K. Mack. Alcohol Involvement in Opioid Pain Reliever and Benzodiazepine Drug Abuse-Related Emergency Department Visits and Drug-Related Deaths - United States, 2010.MMWR Morb. Mortal. Wkly. 63: 881-885 (2014).

[32] S. M. M. Vasconcelos; D. S. Macedo; I. S. P. Lima; F. C. F. Sousa; M. M. F. Fonteles. Cocaetileno: um metabólito da associação cocaína e etanol. Rev. psiquiatr. clín. 28: 207-210 (2001).

[33] N. P. Prior; J. M. Payá; E. S. Company; R. F. Piqueres; M. C. Calvo; R. R. Barco . Trascendencia Del cocaetileno em el consumo combinado de etanol y cocaína. Rev. Esp. De Drogodepend. 31:254-270 (2006).

[34] C. S. Tallarida; K. Bires; J. Avershal; R. J. Tallarida; S. Seo; S. M. Rawls. Ethanol and cocaine: environmental place conditioning, stereotypy, and synergism in planarians. Alcohol (Fayetteville, N.Y.) 48:579-86 (2014).

[35] J. R. Gonçalves. Razões e Implicações das Associações de Álcool ou Maconha com Crack. Tese de Mestrado, Departamento de Saúde Coletiva, Universidade Federal de São Paulo (2013).

[36] A. Romaguera; M. Torrens; E. Papaseit; A. L. Arellano; M. Farré, Concurrent use of cannabis and alcohol: neuropsychiatric effect consequences. CNS Neurol. Disord. Drug Targets 16: $592-597$ (2017).

[37] L. A. Downey; R. King; K. Papafotiou; P. Swann; E. Ogden; M. Boorman; C. Stough. The effects of cannabis and alcohol on simulated driving: influences of dose and experience. Accid. Anal. Prev. 50:879-886 (2013).

[38] J. H. P. van Wel; K. P. C. Kuypers; E. L. Theunissen; S. W. Toennes; D. B. Spronk; R. J. Verkes; J. G. Ramaekers. Single doses of THC and cocaine decrease proficiency of impulse control in heavy cannabis users. Br.J.Pharmacol. 170:1410-1420(2013). 